\title{
Can lung cancer screening by computed tomography be effective in areas with endemic histoplasmosis?
}

\author{
Sandra L. Starnes, MD, ${ }^{a}$ Michael F. Reed, MD, ${ }^{a}$ Cris A. Meyer, MD, ${ }^{b}$ Ralph T. Shipley, MD, \\ Abdul-Rahman Jazieh, MD, ${ }^{\mathrm{c}}$ Elsira M. Pina, DO, ${ }^{\mathrm{d}}$ Kevin Redmond, MD, ${ }^{\mathrm{e}}$ Lynn C. Huffman, MD, ${ }^{\mathrm{a}}$ \\ Prakash K. Pandalai, MD, ${ }^{a}$ and John A. Howington, MD $^{\mathrm{a}}$
}

\begin{abstract}
Objective: Low-dose chest computed tomography (CT) is being evaluated in several national trials as a screening modality for the early detection of lung cancer. The goal of the present study was to determine whether lung cancer screening could be done while minimizing the number of benign biopsy specimens taken in an area endemic for histoplasmosis.
\end{abstract}

\begin{abstract}
Methods: The subjects were recruited by letters mailed to area physicians and local advertisement. The inclusion criteria were age older than 50 years and at least a 20 pack-year smoking history. The exclusion criteria were symptoms suggestive of lung cancer or a history of malignancy in the previous 5 years. The participants completed a questionnaire and underwent a chest $\mathrm{CT}$ scan at baseline and annually for 5 years. The management of positive screening results was determined using a defined algorithm: annual follow-up CT scan for nodules less than $5 \mathrm{~mm}$; 6-month follow-up CT scan for nodules 5 to $7 \mathrm{~mm}$; review by our multidisciplinary tumor board for nodules 8 to $12 \mathrm{~mm}$; and biopsy for nodules greater than $12 \mathrm{~mm}$.
\end{abstract}

Results: A total of 132 patients were recruited. Of the 132 patients, $61 \%$ had positive baseline CT findings and $22 \%$ had positive findings on the annual CT scans. Six cancers were detected. Of these 6 patients, 5 had stage I disease and underwent lobectomy, and 1 had stage IIIA disease and underwent induction chemotherapy and radiotherapy followed by lobectomy. All patients were alive and disease free at a mean follow-up of $41.7 \pm$ 18.6 months. No biopsies were performed for benign lesions. Also, no cancers were missed when the protocol was followed.

Conclusions: Screening with CT can be done effectively in an area endemic for histoplasmosis while minimizing benign biopsies. (J Thorac Cardiovasc Surg 2011;141:688-93)

Lung cancer screening with chest radiography (CXR) was evaluated in the 1970s; however, this did not result in a decrease in mortality in the screened population. ${ }^{1}$ Low-dose chest computed tomography (CT) was introduced in the

From the Division of Thoracic Surgery, Department of Surgery, ${ }^{a}$ Department of Radiology, ${ }^{\mathrm{b}}$ Division of Hematology and Oncology, ${ }^{\mathrm{c}}$ Division of Pulmonary Medicine and Critical Care, Department of Internal Medicine, ${ }^{\mathrm{d}}$ and Department of Radiation Oncology, ${ }^{\mathrm{e}}$ University of Cincinnati College of Medicine, Cincinnati, Ohio.

This work was supported by the University of Cincinnati Barrett Cancer Center.

Disclosures: Sandra L. Starnes is a proctor for video-assisted thoracoscopic surgery lobectomy for Covidien; Michael F. Reed received travel expenses to attend a minimally invasive esophagectomy course paid for by Covidien; Cris A. Meyer has no conflicts of interest to disclose; Ralph T. Shipley has shares in various healthcare companies as a part of his portfolio; Abdul-Rahman Jazieh, Elsira M. Pina, Kevin Redmond, Lynn C. Huffman, and Prakash Pandalai have no conflicts of interest to disclose; John A. Howington is a proctor for video-assisted thoracoscopic surgery lobectomy for Covidien and a member of the Thoracic Advisory Board for Ethicon Endosurgery.

Received for publication Jan 15, 2010; revisions received July 21, 2010; accepted for publication Aug 15, 2010; available ahead of print Oct 8, 2010

Address for reprints: Sandra L. Starnes, MD, Division of Thoracic Surgery, Department of Surgery, University of Cincinnati College of Medicine, 231 Albert B. Sabin Way, PO Box 670558, Cincinnati, OH 45267 (E-mail: sandra.starnes@uc.edu). 0022-5223/\$0.00

Published by Elsevier Inc. on behalf of The American Association for Thoracic Surgery

doi:10.1016/j.jtcvs.2010.08.045 1990s and is currently under evaluation as a lung cancer screening modality., ${ }^{2,3}$ The early results have been promising ${ }^{4}$; however, it has yet to be determined whether screening with low-dose CT will result in decreased mortality.

Pulmonary nodules will be detected in $13 \%$ to $20 \%$ of patients on an initial screening CT scan, ${ }^{2,4}$ with most ultimately diagnosed as benign. One concern about CT screening has been that a large number of screened patients will undergo an invasive procedure for benign lesions. This issue is of particular relevance in areas such as the Ohio River Valley, where histoplasmosis is endemic, resulting in a high incidence of pulmonary nodules in an asymptomatic population. In a CT screening study from the Mayo Clinic, an area with a high prevalence of histoplasmosis, $51 \%$ of baseline screening CT findings were positive. ${ }^{5}$ We undertook the present study to determine the role of lung cancer screening with lowdose CT scan in the Ohio River Valley. We hypothesized that the positive screen rate would be greater than in the large, multicenter national trials. The goals of the present study were to determine the percentage of patients with positive findings on a screening CT scan, the incidence of early cancers detected, and the number of invasive procedures performed for benign lesions. 


\section{Abbreviations and Acronyms \\ $\mathrm{CT}=$ computed tomography \\ $\mathrm{CXR}=$ chest radiography \\ I-ELCAP $=$ International Early Lung Cancer Action Project}

\section{MATERIALS AND METHODS}

The subjects were recruited by letters mailed to community and university physicians, local advertisements, and notices on our institution's multiple cancer Web sites. All participants underwent informed consent, and the protocol was approved by the University of Cincinnati institutional review board. The participants were eligible if they were 50 years old or older and were past or current smokers with at least a 20 pack-year smoking history. The exclusion criteria were symptoms suggestive of lung cancer (eg, new or changing cough, hemoptysis, weight loss, significant dyspnea, or chest wall pain) or a history of malignancy in the previous 5 years (except for nonmelanoma skin cancer). Figure 1 shows the Consolidated Standards of Reporting Trials (Consort) diagram.

The participants completed a short questionnaire concerning demographics, medical history, family history, detailed smoking history, occupational and environmental exposures, and the date and location of any previous CXRs or CT scans. The participants then underwent a low-dose CT scan at baseline and annually for 5 years. All screening CT scans were obtained using a multidetector helical CT scanner (Lightspeed QX/ i 4-slice or Lightspeed Pro 16-slice; General Electric Medical Systems, Milwaukee, Wis) at $120 \mathrm{kV}, 60$ to $65 \mathrm{MA}$, and 5-mm collimation reconstructed to $2.5 \mathrm{~mm}$. The scans were obtained with the participant in the supine position without contrast from the lung apices through the bases using a single inspiratory breath hold. All CT scans were interpreted by 2 chest radiologists (C.M. and R.S.), certified by the American Board of Radiology.

The algorithm used for CT follow-up is shown in Figure 2. If no noncalcified nodules were identified, the screening CT findings were classified as negative. CT scans with 6 or more noncalcified nodules, diffuse bronchiectasis, or ground-glass opacities were classified as showing diffuse disease. The participants with fewer than 6 noncalcified nodules of any size were evaluated according to the defined protocol. If the largest nodule was 4 $\mathrm{mm}$ or smaller, the CT scan was repeated at 1 year. If the largest nodule was 5 to $7 \mathrm{~mm}$, a follow-up CT scan was obtained within 6 months and then annually if no growth had occurred. The cases of patients with nodules measuring 8 to $12 \mathrm{~mm}$ were discussed at our multidisciplinary thoracic on-

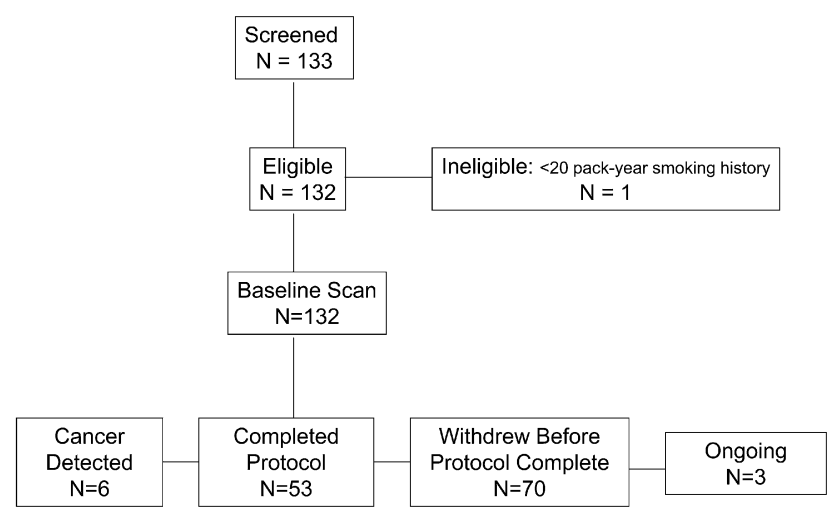

FIGURE 1. Consolidated Standards of Reporting Trials (Consort) diagram.

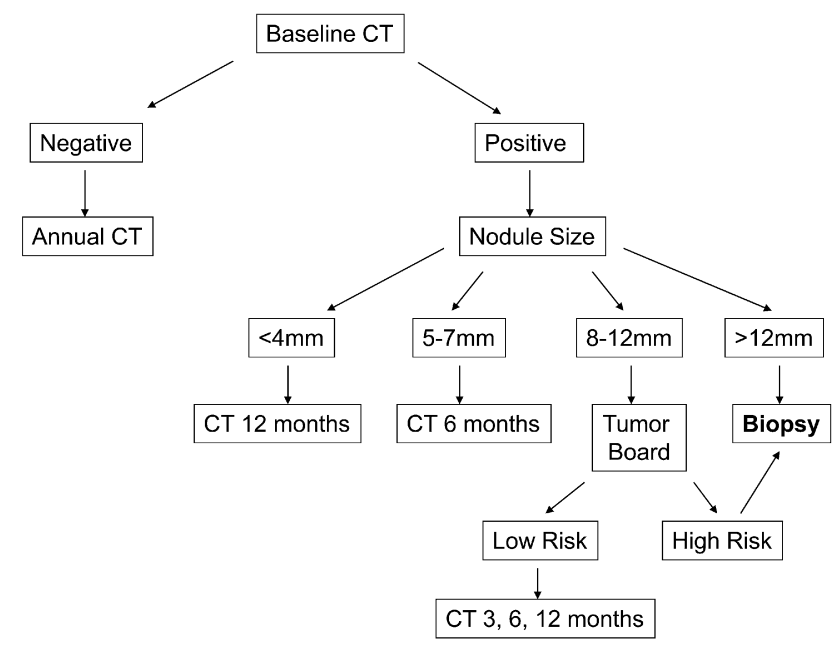

FIGURE 2. Algorithm for computed tomography $(C T)$ follow-up according to nodule size.

cology conference. Participants with nodules that were considered low risk underwent repeat CT scans at 3, 6, and 12 months and then annually if no growth had occurred. Participants with nodules that were considered high risk were recommended to undergo biopsy. The nodules considered high risk were solid and spiculated. Participants with nodules larger than 12 $\mathrm{mm}$ were recommended to undergo biopsy. Biopsy was also recommended for any nodule that was identified as growing on the follow-up CT scans. Biopsy was obtained by bronchoscopy, CT-guided fine needle aspiration, or thoracoscopy with wedge biopsy, depending on the location of the nodule. Positron emission tomography was not a routine part of the algorithm. The participants and their referring physician were notified of the results and the recommendations for any additional evaluation. A referral to a thoracic specialist was provided for participants with a positive screen result.

\section{Statistical Analysis}

Statistical analysis was performed using Statistical Analysis Systems, version 9.1.3 (SAS Institute, Cary, NC). The data are expressed as the mean \pm standard deviation, median and range, or percentages. The number of CT scans between the patients who completed the protocol and those who did not was compared using Student $t$ test. Of the patients who completed the protocol, a $t$ test also was used to compare the number of scans between those in whom nodules were found and those in whom no nodules were found. The nonparametric Spearman rank order correlation was used to determine the relationship between the number of CT scans and nodule size.

\section{RESULTS}

A total of 132 patients were enrolled in the study between August 2001 and July 2005 and underwent a baseline CT scan. The subject characteristics are listed in Table 1. Of the 132 baseline scans, $80(61 \%)$ were considered positive, $49(37 \%)$ were considered negative, and $3(2 \%)$ showed diffuse disease. Of the positive baseline scans, the largest nodule size was 1 to $4 \mathrm{~mm}$ in $43(53.7 \%), 5$ to $7 \mathrm{~mm}$ in $18(22.5 \%), 8$ to $12 \mathrm{~mm}$ in $13(16.3 \%)$ and larger than 12 $\mathrm{mm}$ in 6 patients $(7.5 \%)$. Of the nodules, $75(94 \%)$ were solid, $4(5 \%)$ were ground-glass opacities, and $1(1 \%)$ was partially solid. Of those with positive baseline scan findings without a diagnosis of cancer, the nodules 
TABLE 1. Study subject characteristics

\begin{tabular}{lc}
\hline \multicolumn{1}{c}{ Characteristic } & Value \\
\hline Mean age (y) & $61.5 \pm 5.9$ \\
Gender (n) & \\
$\quad$ Male & $72(54.5 \%)$ \\
Female & $60(45.5 \%)$ \\
Smoking status (n) & \\
$\quad$ Current & $61(46.2 \%)$ \\
Former & $67(50.8 \%)$ \\
$\quad$ Unknown & $4(3.0 \%)$ \\
Mean pack-years smoked & $57.4 \pm 26.7$ \\
\hline
\end{tabular}

remained stable in $60(78 \%)$ and had disappeared on the follow-up scan in $9(12 \%)$. Eight subjects $(10 \%)$ were lost to follow-up.

A total of 496 scans were completed. The number of scans completed per patient is listed in Table 2 . The mean number of scans completed for the entire group was $3.8 \pm 2.2$. Of the 132 subjects, $70(53 \%)$ did not complete the protocol. The number of scans among the group who had completed the protocol compared with the group who had not completed the protocol was $5.6 \pm 1.4$ versus $2.3 \pm 1.4$, respectively $(P<.01)$. Of the patients who completed the protocol, the number of scans obtained for those with nodules versus those without nodules was $5.8 \pm 1.5$ versus $4.6 \pm 0.5$, respectively $(P<.01)$. The number of scans performed did not correlate with the nodule size $(P=.57)$. A total of 31 subjects $(23 \%)$ had new nodules found on an annual CT scan. Of these new nodules, $20(65 \%)$ were solid, $10(32 \%)$ were ground-glass opacities, and $1(3 \%)$ was partially solid. The size was 1 to $4 \mathrm{~mm}$ for $20(65 \%), 5$ to $7 \mathrm{~mm}$ for $6(19 \%)$, 8 to $12 \mathrm{~mm}$ for $1(3 \%)$, and larger than $12 \mathrm{~mm}$ for 4 $(13 \%)$. The nodules remained stable in 17 patients $(55 \%)$ and had disappeared in $6(19 \%)$. Three patients $(10 \%)$ were lost to follow-up, $2(6 \%)$ have continued follow-up, and $3(10 \%)$ were diagnosed as cancer. The 3 patients lost to follow-up all had nodules 1 to $4 \mathrm{~mm}$ in size. The 3 patients with cancer all had nodules larger than $12 \mathrm{~mm}$.

No biopsies were performed for benign lesions. However, 6 patients had nodules larger than $12 \mathrm{~mm}$ and did not undergo biopsy. The first patient had a 30-mm apical nodule on the initial CT scan that had the appearance of an apical scar. She was evaluated by a thoracic surgeon (J.A.H.), and her case was reviewed by the multidisciplinary team and she underwent a positron emission tomography scan, with negative findings. The nodule had remained stable on CT scan 8 months later; however, she withdrew from the study after that point. The second patient had a 15-mm apical nodule. This had the appearance of an apical scar and was followed with serial CT scans and remained stable for 15 months. The patient then withdrew from the study and did not complete the protocol. However, he was alive without apparent disease 8 years after the initial CT scan. The third patient had a $19-\mathrm{mm}$ nodule on the
TABLE 2. Distribution of number of scans per patient

\begin{tabular}{lc}
\hline Scans (n) & Patients (n) \\
\hline 1 & $31(23 \%)$ \\
2 & $12(9 \%)$ \\
3 & $22(17 \%)$ \\
4 & $14(10 \%)$ \\
5 & $22(17 \%)$ \\
6 & $13(10 \%)$ \\
7 & $13(10 \%)$ \\
8 & $5(4 \%)$ \\
\hline
\end{tabular}

initial CT scan. The nodule had a more band-like appearance that was not considered highly suspicious for malignancy. After evaluation by a thoracic surgeon (J.A.H.), he was followed with serial CT scans. The nodule had resolved on the serial CT scans, and he completed the study. The fourth patient had a 13-mm area of ground-glass opacity associated with an area of air space disease on the baseline CT scan. Given the inflammatory appearance, she was followed up with serial CT scans. The ground-glass opacity had resolved and was replaced by an area of band-like scar 1 year later. The fifth patient had had a 30-mm nodule on the baseline CT scan. This had an inflammatory appearance and was recommended to be followed with serial CT scans. She withdrew from the study without undergoing followup; however, a CXR 2 years later was negative, and she was alive without apparent disease 4 years after the original CT scan. The final patient had a 20-mm apical nodule on the initial CT scan. The nodule had the appearance of a scar, and she was recommended to undergo serial CT scans. She withdrew from the study after the baseline CT scan. A chest CT scan performed at an outside institution 6 years after the baseline scan showed a stable nodule. Therefore, despite having nodules larger than $12 \mathrm{~mm}$, none of these 6 patients had been recommended to undergo biopsy after evaluation by a thoracic surgeon in collaboration with the multidisciplinary tumor board.

The median follow-up for the entire cohort was 38 months (range, 0-80 months), and the median nodule size was $4.0 \mathrm{~mm}$ (range, 3-30 mm). The median follow-up for subjects with a positive screening CT scan was 48.5 months (range, 0-80 months), and the average number of scans was $4.3 \pm 2.1$. Of the 132 patients, 70 did not complete the entire protocol. Of these 70 patients, 27 had nodules found but did not complete the recommended follow-up from the screening protocol. However, we were able to obtain imaging results from studies performed outside of the protocol for 14 of the 27 patients. The nodules of all of these 14 patients remained stable. Of the 70 patients who did not complete the protocol, 13 had not even completed the 2-year follow-up examination for their nodules.

A total of 6 cancers were detected, representing $4.5 \%$ of the study population (Table 3 ). Three patients had lung 
TABLE 3. Clinical and pathologic characteristics of patients diagnosed with lung cancer

\begin{tabular}{llcccccc}
\hline Pt. No. & Gender & Age (y) & $\begin{array}{c}\text { Pack-years } \\
\text { smoked }\end{array}$ & Clinical stage & Treatment & Pathologic stage & Histologic finding \\
\hline 1 & Female & 69 & 51 & IA (T1N0M0) & VATS lobectomy & IA (T1N0M0) & Adenocarcinoma \\
2 & Male & 57 & 78 & IA (T1N0M0) & VATS lobectomy & IA (T1N0M0) & Adenocarcinoma \\
3 & Female & 58 & 40 & IA (T1N0M0) & VATS lobectomy & IB (T2N0M0) & Adenocarcinoma \\
4 & Female & 55 & 44 & IA (T1N0M0) & Lobectomy & IA (T1N0M0) & Adenocarcinoma \\
5 & Male & 61 & Unknown & IA (T1N0M0) & VATS lobectomy & IB (T2N0M0) & Adenocarcinoma \\
6 & Male & 60 & 64 & IIIA (T2N2M0) & Chemotherapy, radiotherapy, & IB (T2N0M0) & Squamous \\
\end{tabular}

VATS, Video-assisted thoracoscopic surgery.

cancer diagnosed on the baseline CT scan and 3 had cancer diagnosed from a subsequent annual CT scan. All 3 interval cancers were the result of new nodules seen on the interval scan that were recommended to undergo biopsy and not the growth of a previously detected nodule. The mean nodule size at the diagnosis of cancer was $19.5 \mathrm{~mm}$ (range 8-53 $\mathrm{mm}$ ). Of the 6 cancers, 5 were in solid nodules and 1 was in a partially solid nodule. All patients underwent biopsy, which confirmed malignancy, before treatment: 1 with CT-guided fine needle aspiration, 1 with bronchoscopy, and 4 with video-assisted thoracoscopic surgery wedge biopsy performed at the planned resection. All patients with lung cancer underwent surgical treatment. Of the 6 patients, 5 underwent lobectomy and mediastinal lymph node dissection as the only treatment; 3 had pathologic stage IA disease and 2 had pathologic stage IB. The preoperative diagnosis for the sixth cancer was stage IIIA disease. That patient underwent induction chemotherapy and radiotherapy followed by lobectomy. The mean follow-up time was $41.7 \pm 18.6$ months for these 6 patients, and all 6 were alive without recurrence at the last follow-up visit.

Two other patients in the cohort were diagnosed with lung cancer. One patient had negative baseline CT scan findings. She withdrew from the study and was diagnosed with stage IV spindle cell lung cancer 36 months after her initial CT scan. She was treated with chemotherapy but died of lung cancer 6 months after the diagnosis. Another patient was found to have a $3-\mathrm{mm}$ nodule on the initial CT scan. He withdrew after his baseline CT scan, and stage IV small cell cancer was diagnosed 16 months later. He underwent chemotherapy but ultimately died of his disease.

The cost per scan was $\$ 150$, for a total cost to the project of $\$ 73,500$. The cost per cancer detected was $\$ 12,250$.

\section{DISCUSSION}

Screening for malignancies such as breast and colon is currently recommended to detect these cancers early and thereby decrease mortality. However, although lung cancer is the number 1 cause of cancer mortality, currently screening is not recommended. ${ }^{6}$ The Como International Conference held in 2003 reviewed the current evidence for screening for the early detection of lung cancer. Although widespread screening was not advised, it was recommended that physicians should discuss the available options for lung cancer screening and the risks and benefits involved with those at high risk of lung cancer. ${ }^{7}$

The screening trials in the 1970s using CXR did not demonstrate a reduction in mortality in the screened population. The Mayo Lung Project randomized more than 9000 male smokers to CXR and sputum cytology every 4 months versus the usual care. ${ }^{1}$ The recommendations at that time included a yearly CXR and sputum cytology. That trial failed to show a decrease in lung cancer mortality. However, the trial has been criticized for an inadequacy of power and contamination with the nonscreened group. Of the subjects in the observation group, $72 \%$ had undergone a CXR during the study period, because that was standard care at that time.

Low-dose chest CT was introduced in the 1990s as a screening modality. Several recent trials have shown promise for this method in detecting early-stage lung cancer. The National Cancer Institute published the results of a feasibility trial of lung cancer screening with low-dose chest CT in 2004. A total of 3318 current and former smokers were randomized to low-dose chest CT versus CXR as a one time screening test. Positive results were detected in $20.5 \%$ in the chest CT arm and $9.8 \%$ in the CXR arm. Lung cancer was detected more often in the subjects randomized to low-dose CT $(1.9 \%$ versus $0.45 \%)$. Of the subjects with positive CT findings, lung cancer was diagnosed in $9.2 \%$. In $8 \%$ of subjects with a positive screen result, an invasive procedure was performed that revealed a benign process. ${ }^{2}$ Because of that feasibility trial, the National Lung Screening Trial was launched, with the goal of randomizing 50,000 subjects to annual screening with low-dose chest CT versus CXR. The trial is currently ongoing.

The International Early Lung Cancer Action Project (I-ELCAP) recently reported the results of CT screening in more than 30,000 subjects. ${ }^{4}$ A positive baseline CT screening result was detected in $13 \%$ of the subjects. Overall, biopsy for a benign lesion occurred in $8 \%$ of subjects with a positive screen result and $0.14 \%$ of the subjects overall. Using that screening protocol, lung cancer was diagnosed in 484 patients, and $85 \%$ of those patients had clinical stage I disease. The overall 10 -year survival rate 
for all patients diagnosed with lung cancer was $80 \%$, a significant improvement compared with the current $15 \% 5$ year survival rate.

If the National Lung Screening Trial and the I-ELCAP trial show a decrease in lung cancer mortality, screening with low-dose chest CT will likely become widespread. However, most patients with a positive screen result will not have lung cancer. One concern with CT screening for lung cancer is the morbidity and expense of the invasive procedures for benign lesions. Although many have accepted a moderate degree of benign breast biopsy findings for breast cancer, biopsies of pulmonary lesions have been deemed more invasive. Therefore, the number of invasive procedures performed for benign lesions should be minimized. In the 2 largest screening trials, the baseline CT scan results were positive for $13 \%$ to $20 \%$ of subjects. ${ }^{2,4}$ In some areas of the United States such as the Ohio River Valley, we can expect a greater rate of positive screening CT results because of the endemic histoplasmosis. We undertook the present study to define the rate of positive baseline screening CT results in a geographic area of endemic histoplasmosis and to determine whether lung cancer screening would be feasible while limiting the number of biopsies performed for benign processes. We have shown that the rate of positive screening CT results was $61 \%$, triple the rate of most previous studies. The number of positive annual screen results $(22 \%)$ was also significantly greater than in the I-ELCAP trial. ${ }^{3}$ We did not use a size cutoff to define a positive nodule, because any nodule would need to be followed up by our defined protocol. However, some screening studies such as the IELCAP study used a size cutoff of $5 \mathrm{~mm}$ to define a positive study. ${ }^{4}$ If we had used a 5 -mm cutoff, $28 \%$ of our baseline scans would have been recorded as positive, still greater than that reported by most screening studies. Our findings are similar to the CT screening trial performed at Mayo Clinic, another area endemic for histoplasmosis, in which $51 \%$ of participants had a positive baseline screening CT result and $74 \%$ of participants had positive baseline or annual scan results. ${ }^{8}$ That study also defined any nodule, irrespective of size, as a positive result, and $61 \%$ of nodules in their study were less than $4 \mathrm{~mm}$, comparable to our findings.

Despite the high rate of positive screening results in the present study, no invasive procedures were performed for benign disease. However, 6 subjects had nodules greater than $12 \mathrm{~mm}$ that were not recommended for biopsy. According to our protocol, these nodules should have been referred for biopsy. However, all these nodules were thought to be likely benign after review by a dedicated general thoracic surgeon in conjunction with the multidisciplinary thoracic tumor board. Although many of these 6 patients did not complete the recommended protocol, longer follow-up confirmed that all the nodules were likely benign. Two of the nodules had resolved on subsequent scans. The other nod- ules were stable 8 months to 6 years later. Given these findings, future screening protocols at our institution will be revised to include thoracic tumor board review and/or review by a dedicated thoracic oncology specialist for nodules larger than $12 \mathrm{~mm}$.

These results have confirmed that even in an area with a high prevalence of pulmonary nodules, CT screening can be performed with a very low number of biopsies needed for benign lesions. However, if CT screening does show a decrease in lung cancer mortality and becomes the standard of care, we must exercise caution in recommending invasive procedures. We, and others, have shown that a low rate of benign biopsies is possible in a defined setting. First, established protocols must be followed by the participants' referring physicians. Biopsy specimens taken outside of the defined protocol are more likely to be benign. ${ }^{3}$ Second, not every nodule larger than $12 \mathrm{~mm}$ must be biopsied immediately. We have shown that some of these nodules will be inflammatory and can be followed safely through involvement of a dedicated thoracic oncology specialist, such as a general thoracic surgeon or a pulmonologist, in collaboration with a multidisciplinary thoracic oncology team.

In the present study, the lung cancer detection rate was $4.5 \%$ of the screened population, slightly greater than that in previous studies, in which the rate was 1.3 to $2.7 \% .^{2-4}$ Similar to other trials, 5 of our 6 patients with cancer had stage 1 lung cancer. All patients underwent surgery, and all were alive and free of cancer recurrence at the latest follow-up visit.

One limitation of our study was the high number of patients who did not complete the protocol, with more than $50 \%$ of our patients not undergoing all $5 \mathrm{CT}$ scans. The compliance was lower than that in other studies such as the Mayo Clinic screening trial, which had $80 \%$ compliance at 4 years. ${ }^{8}$ The level of compliance remains to be seen if CT screening becomes the standard of care. A part of the reason for our lower compliance might have been that the participants paid (\$150) for each CT scan with normal findings. The follow-up CT scans after a positive result were covered by the insurance companies, and we had funding (University of Cincinnati Cancer Center) for the participants who could not afford the fee. The compliance rate might have been better if the scans had been free or covered by insurance.

The primary limitation of the present study was the small number of patients. However, the goal of the present trial was not to repeat the goals of the large national trials. Rather, our goal was to determine whether successful screening with a low rate of benign biopsy findings would be possible despite the greater rate of lung nodules occurring in the Ohio River Valley population. However, it is unclear whether these results would be reproducible in a large number of patients.

In the present study, we detected a similar rate of earlystage lung cancers without performing invasive procedures 
for benign lesions. The development of a defined protocol for screening and the participation of a multidisciplinary lung cancer team in the current study likely contributed to the avoidance of invasive procedures for benign lesions despite the high incidence of pulmonary nodules. If lung cancer screening is adopted nationally, a similar approach would likely contribute to keeping the number of invasive procedures for benign lesions to an acceptably low rate.

We would like to thank Laura James, MS, for her assistance with the statistics.

\section{References}

1. Marcus PM, Bergstralh EJ, Fagerstrom RM, Williams DE, Fontana R, Taylor W, et al. Lung cancer mortality in the Mayo Lung Project: impact of extended followup. J Natl Cancer Inst. 2000;92:1308-16.
2. Gohagan J, Marcus P, Fagerstrom R, Pinsky P, Kramer B, Prorok P. Baseline findings of a randomized feasibility trial of lung cancer screening with spiral CT scan versus chest radiograph. Chest. 2004;126:114-21.

3. Henschke C, McCauley D, Yankelevitz D, Naidich DP, McGuinness G Miettinen OS, et al. Early lung cancer action project: overall design and findings from baseline screening. Lancet. 1999;354:99-105.

4. The International Early Lung Cancer Action Program Investigators. Survival of patients with stage I lung cancer detected by CT screening. $N$ Engl J Med. 2006;355:1763-71.

5. McMahon PM, Kong CY, Johnson BE, Weinstein MC, Weeks JC, Kuntz KM, et al Estimating long-term effectiveness of lung cancer screening in the Mayo CT screening study. Radiology. 2008;248:278-87.

6. Bach PB, Silvestri GA, Hanger M, Jett JR. Screening for lung cancer: ACCP evidence-based clinical practice guidelines (2nd edition). Chest. 2007;132: 69S-77.

7. Strauss GM, Dominioni L, Jett JR, Freedman M, Grannis FW. Como International Conference Position Statement: lung cancer screening for early diagnosis 5 years after the 1998 Varese conference. Chest. 2005;127:1146-51.

8. Swensen SJ, Jett JR, Hartman TE, Midthun DE, Mandrekar SJ, Hillman SL, et al. CT screening for lung cancer: five-year prospective experience. Radiology. 2005; 235:259-65. 